\title{
Intermediate Band Solar Cells: Present and Future
}

\author{
I. Ramiro* and A. Martí \\ Instituto de Energía Solar, Universidad Politécnica de Madrid, 28040 Madrid, Spain
}

\begin{abstract}
In the quest for high-efficiency photovoltaics (PV), the intermediate band solar cell (IBSC) was proposed in 1997 as an alternative to tandem solar cells. The IBSC offers $63 \%$ efficiency under maximum solar concentration using a single semiconductor material. This high efficiency limit attracted the attention of the PV community, yielding to numerous intermediate band (IB) studies and IBSC prototypes employing a plethora of candidate IB materials. As a consequence, the principles of operation of the IBSC have been demonstrated, and the particularities and difficulties inherent to each different technological implementation of the IBSC have been reasonably identified and understood. From a theoretical and experimental point of view, the IBSC research has reached a mature stage. Yet, we feel that, driven by the large number of explored materials and technologies so far, there is some confusion about what route the IBSC research should take to transition from the proof of concept to high efficiency. In this work, we give our view on which the next steps should be. For this, first we briefly review the theoretical framework of the IBSC, the achieved experimental milestones, and the different technological approaches used, with special emphasis in those recently proposed.
\end{abstract}

\section{KEYWORDS}

Intermediate band, high efficiency, solar cell

\section{*Correspondence:}

Iñigo Ramiro, Instituto de Energía Solar, Universidad Politécnica de Madrid, 28040 Madrid, Spain

E-mail: i.ramiro@ies.upm.es 


\section{INTRODUCTION AND CONTEXT}

The intermediate band solar cell (IBSC) was proposed by Luque and Martí ${ }^{1}$ as a structurally simple yet highly efficient photovoltaic (PV) concept. It builds on and completes an early idea by Wolf $^{2}$ of exploiting in-gap levels to allow bellow-bandgap photon absorption as a means of surpassing the efficiency limit for conventional single-gap solar cells (SGSC), known as the Shockley and Queisser (S\&Q) limit. ${ }^{3}$ To summarize the basis and operation of the IBSC we will rely on Figure 1a.

The $\mathrm{S} \& \mathrm{Q}$ limit imposes a maximum conversion efficiency -determined only by the bandgap, $E_{G}$, of the absorbing material- under the assumption that all photons with energy higher than $E_{G}$ are sub-optimally harvested (because of carrier thermalization), and all photons with energy lower than the bandgap are wasted (not absorbed). The IBSC reduces non-absorption losses by introducing the idea of an intermediate band (IB) material. The optoelectronic properties of such material, similarly to a semiconductor, are defined by three electronic bands: the conventional valence and conduction bands (VB and CB) and an additional band, the IB, that lies in-between those two (in Figure 1a the IB is arbitrarily placed closer to the VB). Part of the photons with energy lower than $E_{G}$ can be absorbed in electronic transitions from the VB to the IB (transition 1 in the figure) and from the IB to the $\mathrm{CB}$ (transition 2). These two additional sub-gaps are generally named $E_{H}$ and $E_{L}$, for the higher one and the lower one, respectively. In our description, the energy width of the IB will be considered approaching zero so that optical and electronic gaps have the same values and $E_{G}=E_{H}+E_{L}$. Removing this condition leads to interesting variations of the IBSC concept such as the so-called ratchet IBSC., ${ }^{4,5}$

Extra electron-hole pairs are generated via a two-photon absorption process, using the IB as steppingstone, which yields to an increase in photocurrent. Despite the contribution of subbandgap photons to the photocurrent, the maximum voltage that an ideal IBSC can deliver is fundamentally limited by $E_{G}$, and not the sub-gaps $E_{H}$ or $E_{L}$. This phenomenon is usually called voltage preservation and demands that non-radiative channels connecting the IB and the other two bands, such as Auger or phonon-assisted recombination, are minimized. For this reason, an ideal IB material is usually described as having a null density of states in between the IB and the other two bands, which hampers phonon-assisted recombination. The time scale of intraband electron-electron interaction processes within each band is assumed to be much shorter than interband electron-electron processes (for example between the CB and the IB) and therefore, the carrier population in each band is described by its own electrochemical potential or quasi-Fermi level: $\mu_{C}, \mu_{V}$, and $\mu_{I}$, for the $\mathrm{CB}, \mathrm{VB}$ and IB, respectively. In addition all the electrons are assumed to interact with a common background of photons and phonons so that all these particles: electrons (independently of the band where they are), photons and phonons share the same temperature (say, room temperature $T_{C}$ ). ${ }^{6,7}$ 
In an ideal IBSC, with high carrier mobility, the output voltage $e \cdot V$, where $e$ is the elementary charge, is equal to the electrochemical potential difference $\mu_{C}-\mu_{V}$ and is independent of $\mu_{I}$. To ensure this, it is necessary to include in the device hole and electron selective contacts (HSC and ESC) that allow extracting electrons from the $\mathrm{CB}$ (current $J_{e}$ ) and holes from the VB (current $J_{h}$ ), but not from the IB (Figure 1a).

(a)

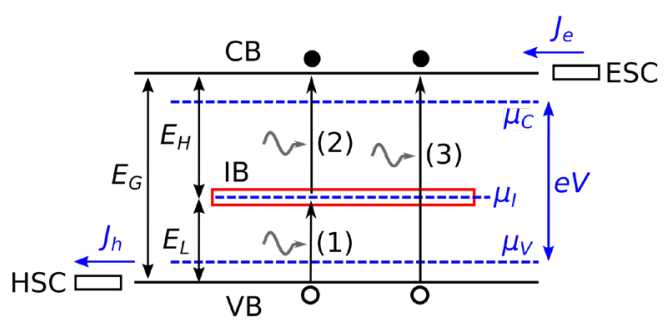

(b)

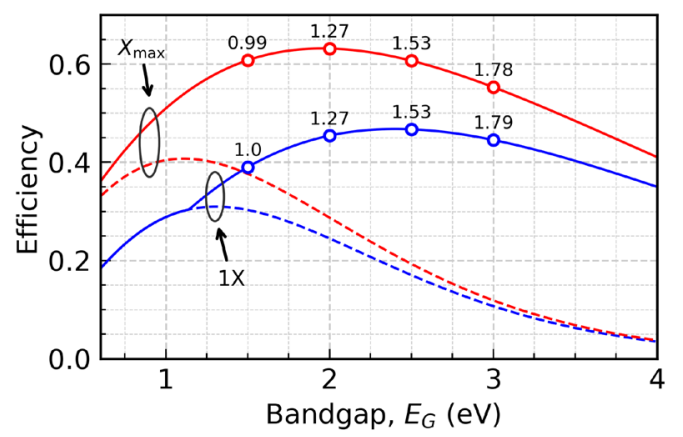

(c)

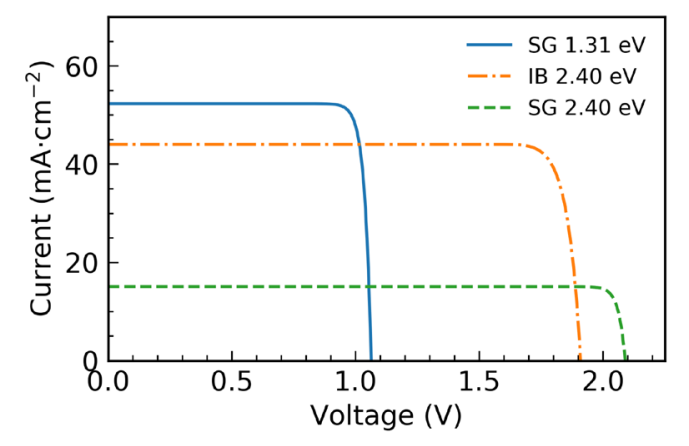

FIGURE 1. (a) Sketch of the simplified band diagram and operation of an IBSC. (b) Limiting efficiency of an ideal SGSC (broken lines) and an ideal IBSC (solid lines) as a function of $E_{G}$. Red lines represent the case of maximum sunlight concentration $\left(X_{\max }\right)$, whereas blue lines represent one-sun illumination (1X). The value of $E_{H}$ that maximizes the efficiency of the IBSC is indicated for some values of $E_{G}$. (c) $J-V$ characteristic under one-sun illumination of an ideal SGSG with optimum bandgap (1.31 eV), an ideal IBSC with optimum bandgap ( $2.40 \mathrm{eV})$, and an ideal SGSC with bandgap $2.40 \mathrm{eV}$.

Thanks to the presence of the IB and the carrier selective contacts, IBSCs can achieve efficiencies as high as $63 \%{ }^{1}$ under maximum light concentration (see Figure $1 \mathrm{~b}$ ), which represents a relative increment of around $50 \%$ with respect to conventional SGSCs. ${ }^{3}$ Actually, the limiting efficiency of an IBSC is very close to that of a tandem cell with three gaps. ${ }^{8}$ The potential high efficiency, combined with a conceptually simple structure, for instance, when compared with multi-junction solar cells (MJSC), were probably decisive factors that motivated extensive research on the topic. ${ }^{9,10}$ Many different IB materials have been explored, as we will discuss later on. Some of them implied expensive raw materials and/or fabrication methods, but the prospect of high 
efficiency and relatively small cells used in concentration PV (CPV) systems made the research worthwhile, not only scientifically, but also from the point of view of the energy price. ${ }^{11}$ However, the PV landscape has changed greatly in the las two decades. On the one hand, the price of flat panel Si PV has experienced a major decrease as the annual installed capacity increased. ${ }^{12}$ On the other hand, MJSCs are established as a valid technology for CPV systems, with demonstrated efficiencies well over $40 \%,{ }^{13}$ depending on the number of junctions, and present in the industry. ${ }^{14}$ In this new context, it is worth recalling that, although less frequently pointed out, the IBSC concept is equally powerful under one-sun illumination (Figure 1b), in the sense that it can exceed the SGSC efficiency limit by around 50\%. ${ }^{15}$ The idea of an IBSC working at one sun entails some changes in the design and fabrication of IB materials and devices. Firstly, the bandgap of a highly efficient IBSC depends on the sunlight concentration factor. Under maximum concentration, the limiting efficiency is higher than $60 \%$ in the range $1.5 \mathrm{eV}<E_{G}<2.5 \mathrm{eV}$, being $1.96 \mathrm{eV}$ the optimum value. However, at one-sun, the efficiency is higher than $40 \%$ for $1.5 \mathrm{eV}<E_{G}<3.5 \mathrm{eV}$, being $2.40 \mathrm{eV}$ the optimum value. This opens the possibility of exploring wide-bandgap materials, with $E_{G}>2.5 \mathrm{eV}$, as high-efficiency IB absorbers. Secondly, the cost of the employed materials for solar cell manufacturing gains importance in PV systems working at one sun vs concentration systems and needs to be more carefully considered.

Figure 1c plots the current-voltage $(J-V)$ characteristics of an ideal SGSC and an ideal IBSC with optimum bandgaps working at one sun. When compared with the optimum SGSC, the IBSC exhibits somewhat less photogenerated current but a larger voltage, which combined yield to an increased output power. It is also illustrative to compare the curve of the optimum IBSC with an ideal SGSC having the same bandgap $(2.40 \mathrm{eV})$. The SGSC delivers higher output voltage but a much lower current, consequence of the lower number of high-energy photons in the solar spectrum. This example serves to clarify the concept of voltage preservation in IBSCs. Voltage is said to be preserved when it is not limited by the sub-gaps introduced by the IB, this is, when $e \cdot V>E_{H}$. This does not mean that the open-circuit voltage $V_{O C}$ is not reduced upon the inclusion of the IB when compared to a SGSC with the same total gap but without the IB. In fact, under sunlight concentration smaller than $X_{\max }$, the inclusion of the IB entails a reduction of $V_{O C}$ as compared to the ideal SGSG with the same gap, as shown in Figure 1c, but the gain in current is such that the output power balance lies in favor of the IBSC. The reason for this reduction in $V_{O C}$ is the extra recombination channels -even if radiative-introduced by the IB, which are dominant at low sunlight concentration.

The solar cell efficiencies and $J-V$ curves previously discussed were obtained from detailed balance calculations ${ }^{1,3}$ for a solar cell operating at $300 \mathrm{~K}$, modelling the sun as a blackbody at $6000 \mathrm{~K}$, and setting $X_{\max }=46050$ suns. Higher efficiency values are obtained if the AM1.5D tabulated spectrum is considered. ${ }^{16}$ It has also been assumed that the absorption coefficients of 
the three bands do not overlap, which ensures that each photon is absorbed in the largest possible transition and yields the highest efficiency in the optimum case. The removal of the constraint of non-overlapping absorption coefficients results in different efficiency values and can be beneficial when the IB is not placed at the optimum position. ${ }^{15,17,18}$

\section{TECHNOLOGICAL APPROACHES EMPLOYED IN IBSC}

The different technological approaches employed so far to manufacture IB materials and IBSC prototypes can be grouped in four categories, summarized in Table 1 and illustrated in Figure 2ad. (a) Quantum dots (QDs). The IB stems from confined states of the QDs. ${ }^{19}$ In this work we will differentiate between two QD technologies, epitaxial QDs and colloidal QDs, since the use of one or the other may come with important practical differences, as we will discuss later on. (b) Bulk with deep-level impurities (DLIS). In this approach, the IB is formed by the deep levels introduced by impurities in a host material..$^{20}$ There is controversy, though, about whether an IB emerging from a high density of deep levels will be actually able to suppress non-radiative recombination, ${ }^{21}$ a necessary condition for high efficiency. (c) Highly mismatched alloys (HMAs). In this kind of alloys, the inclusion of a small fraction of a new element in the host, interacts with one the bands (the $\mathrm{CB}$ in the illustration) of the host, splitting it into two sub-bands, $E_{+}$and $E_{-.}{ }^{22}$ The least energetic sub-band $\left(E_{-}\right)$is taken as the IB. ${ }^{23}$ (d) Organic molecules (OMs). This approach makes use of different organic species that play the role of either sensitizer or high-bandgap acceptor. ${ }^{24}$ The sensitizer molecules can absorb photons with energy lower than the bandgap $E_{G}$ of the acceptor, transitioning from the ground state to an excited singlet state. This singlet state can naturally relax into a triple state of the same species. Subsequently, a process of energy transfer (ET) between the sensitizers and the acceptor can take place, leading to triplet states in the acceptor. Finally, two triple states in acceptor molecules can combine and give raise, via a triplettriplet annihilation (TTA) process, to one higher-energy singlet state of the acceptor species. In essence, the two below-bandgap photons absorbed in the sensitizers are up-converted ${ }^{25}$ into one high-energy electron-hole pair in the high-energy absorber. The reader is referred to Refs. ${ }^{24}$ and

${ }^{25}$ for more detailed explanation of this mechanism.

In addition to these approaches, inspired perhaps by some physical intuition, there has been extensive theoretical work based on first-principles calculations as a way of verifying or predicting the existence of an IB in a given alloy (for example, $\mathrm{V}$ in $\operatorname{In}_{2} \mathrm{~S}_{3},{ }^{26}$ perovskite based systems, ${ }^{27} \mathrm{ZnS}$ and $\mathrm{ZnTe},{ }^{28} \mathrm{CdSe}$ nanoparticles, ${ }^{29}$ or (N, P, As and $\mathrm{Sb}$ ) doped $\mathrm{Cu}_{2} \mathrm{ZnSiSe}_{4}{ }^{30}$ ). 
(a)

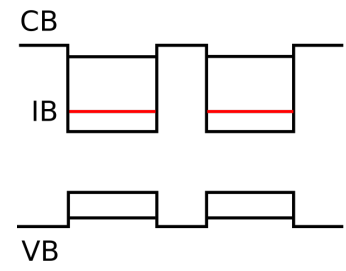

(c)

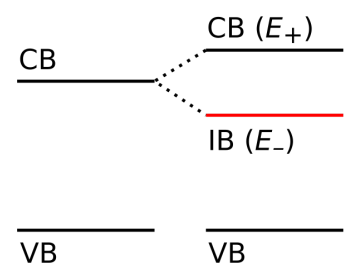

(b)

$\mathrm{CB}$

IB

VB

FIGURE 2. Simplified band diagram of the different technological approaches used in IBSCs. (a) Quantum dots. (b) Bulk with deep level impurities. (c) Highly mismatched alloys. (d) Organic molecules. For consistency in the nomenclature, the highest occupied molecular orbital (HOMO) and the lowest unoccupied molecular orbital (LUMO) of the high-bandgap molecular absorber are identified, respectively, as the VB and the CB.

\begin{tabular}{|c|c|c|}
\hline Technological approach & Origin of the IB & $\begin{array}{c}\text { Proposed for IBSC / } \\
\text { First employed }\end{array}$ \\
\hline \hline Quantum dots (QDs) & Confined levels in the quantum dots & $2000^{19} / 2004^{31}$ \\
\hline Bulk with deep-level impurities (DLIs) & Levels introduced by the impurities & $2001^{20} / 2012^{32}$ \\
\hline Highly mismatched alloys (HMAs) & Split of the CB or the VB of the alloy & $2003^{23} / 2009^{33}$ \\
\hline Organic molecules (OMs) & Singlet and triplet molecular states & $2008^{24} / 2015^{34}$ \\
\hline
\end{tabular}

TABLE 1. Technological approaches employed in IBSC fabrication.

\section{EXPERIMENTAL MILESTONES \& TECHNOLOGY STATUS}

\subsection{Achieved and pending experimental milestones}

Some of the most relevant achieved experimental milestones in IBSC research are sorted in chronological order in Figure 3. Additionally, the emergence of IBSC technological approaches is also indicated. As described before, an IBSC should produce current when illuminated with two below-bandgap photons that promote electrons from the VB to the IB and from the IB to the CB. This process of two-photon photocurrent (TPPC) was first demonstrated in 2006 using InAs/GaAs EQDs operating at low temperature..$^{35}$ Initially, these photocurrent experiments were 
taken using broadband infrared light. It took almost one decade more to achieve energy spectral resolution in the TPPC, in $\operatorname{In}(\mathrm{Ga}) \mathrm{As} / \mathrm{AlGaAs}$ EQD prototypes operating at low temperature. ${ }^{36,37}$ It is important to remark that an ideal IBSC, without overlapping in the absorption coefficients, should not produce photocurrent under monochromatic below-bandgap illumination. However, as introduced earlier, some degree of overlapping may be beneficial in practice for some cases in which the IB is placed in a sub-optimal position. Additionally, the existence of other non-radiative processes such as thermal or tunnel electron exchange between the IB and the $\mathrm{CB}$ or $\mathrm{VB},{ }^{38}$ or Auger generation in one of the sub-gaps ${ }^{39}$ may lead to photo-response to monochromatic belowbandgap illumination even in the case of non-overlapping absorption coefficients. Monochromatic below-bandgap photocurrent was the first signature of an optically active IB in early EQD-based IBSC prototypes ${ }^{31}$ and is still today one of the first IB signatures investigated in new devices.

The first demonstration of voltage preservation $\left(V_{O C}>E_{H} / e\right)$ was reported in InAs/GaAs EQD prototypes operating at low temperature in $2010 .{ }^{40} \mathrm{~A}$ step forward was given recently with the demonstration in $\mathrm{GaSb} / \mathrm{GaAs}$ EQD prototypes, also at low temperature, of two-photon photovoltage, ${ }^{41}$ that is, that two-step two-photon below-bandgap absorption produces an increase in photovoltage with respect to one-photon below-bandgap absorption. Finally, the existence of three electrochemical potentials in the IBSC comes with a luminescence signature with three distinct emission peaks corresponding to the three gaps of the IB material. ${ }^{42}$ This characteristic IBSC signature was first reported in GaNAs HMA prototypes in 2011 via electroluminescence measurements at low temperature. ${ }^{43}$

In our view, two main experimental milestones are still pending. The first one is the simultaneous demonstration of photocurrent response to below-bandgap photons and voltage preservation. In this respect, so far, below-bandgap absorption has been reported under short-circuit conditions $(V$ $=0)$, and voltage preservation has been reported at open circuit $(J=0)$. In both cases the power delivered by the cell is zero. The production of below-bandgap photocurrent when the cell is producing power, and specifically when $e \cdot V>E_{H}$, would be a necessary condition for the second and more demanding milestone: the demonstration of an increase in the cell efficiency, which will finally lead to high-efficiency devices. Finally, it is worth noting that some of the discussed milestones have been obtained generally under cryogenic temperatures. The ultimate goal, of course, is achieving a practical IBSC, which would require that all the previously mentioned phenomena take place at room temperature. 


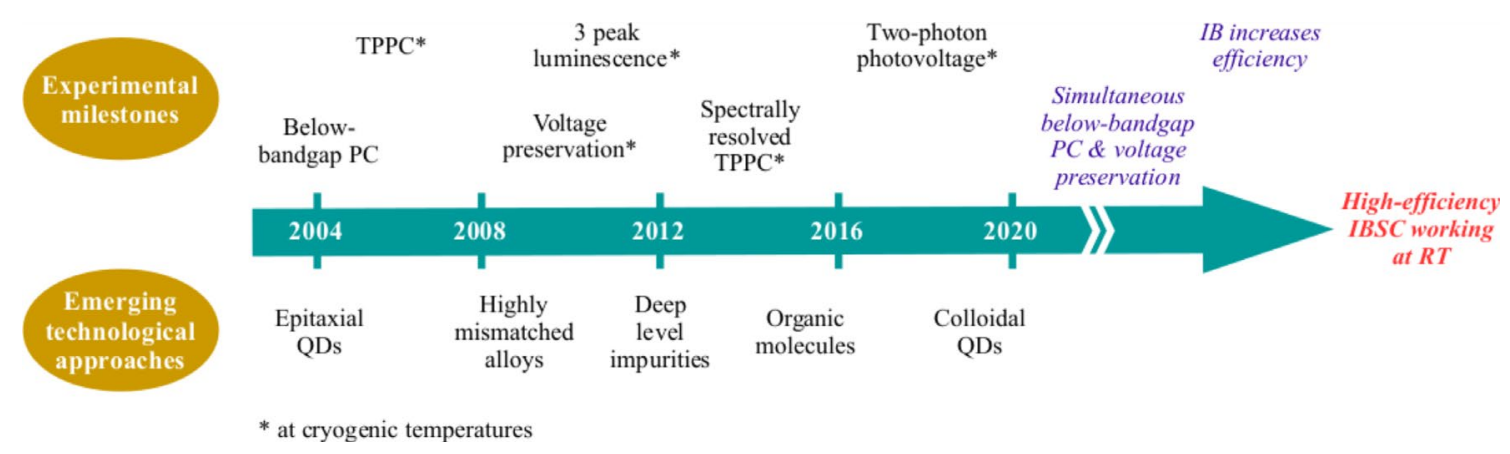

FIGURE 3. Experimental progress in IBSC development from the perspectives of achieved experimental milestones and the demonstration of new technological approaches. In purple, milestones yet to be achieved. In red, the ultimate goal: a practical high-efficiency IBSC.

\subsection{IBSC technology status}

Although experimental progress has been made within each technological approach, none of the IBSC implementations so far have fully exploited the benefits of the IB.

The use of OMs in IB devices is still in its infancy, yet demonstration of below-bandgap photocurrent in the first reports gives an indication of its potential. ${ }^{34,44,45}$ Research is needed to find the adequate combination of sensitizers and acceptors for which the ET and TTA processes are efficient, paying attention to how this process is affected by the operation voltage of the cell. Bulk semiconductors with DLIs have demonstrated the capability of achieving relatively strong below-bandgap photocurrent. ${ }^{32,46}$ New candidate materials continue to be proposed and analyzed, ${ }^{47-54}$ generally proving below-bandgap absorption, which evidences that the DLI approach is far from exhausted. However, we think that at this moment more profound studies are needed. It is important to discriminate IB candidates based on the amount of non-radiative recombination introduced by the deep levels, which will ultimately determine whether the IB plays a detrimental or beneficial role. In this regard, Ref. ${ }^{55}$ presents a model for predicting the suitability of an IB candidate material from basic materials properties.

In a similar line, HMAs have proven its potential as below-bandgap absorbers; $;^{33,43,56,57}$ but studies aimed to understand how to preserve the voltage are still lacking and should be addressed.

QDs, in particular epitaxial quantum dots (EQDs), are the most investigated IB technology ${ }^{9}$ and the one that has allowed verification of the underlying physics of the IBSC, as previously detailed. Nevertheless, EQD-based IBSCs face two major problems. First, absorption of the transitions involving the IB is too weak, mainly due to the low volumetric concentration of EQDs (in the order of $10^{15}-10^{16} \mathrm{~cm}^{-3}$ ). As an example, Figure 4 shows photocurrent produced in an InAs/AlGaAs EQD-based IBSC $^{58}$ where below-bandgap photocurrent is several orders of magnitude weaker than supra-bandgap photocurrent. Similar behavior is obtained in other EQD 
systems such as $\mathrm{GaSb} / \mathrm{GaAs} .{ }^{59}$ To enhance absorption in the QD material, light trapping techniques such as texturing ${ }^{60,61}$ or plasmonic scattering ${ }^{62}$ have been investigated, although the results are still far from the requirements of a high-efficiency IBSC. ${ }^{63}$ The second problem is excessive non-radiative electron exchange between the IB and the VB or the CB of the host, which prevents the preservation of the voltage at room temperature. ${ }^{38,64}$ This fast electron exchange is due to the non-optimal size and shape of EQDs, which give rise to closely spaced confined electronic levels, favoring carrier thermalization; and/or to electron-hole Auger recombination, which may be dominant in type-I EQDs. ${ }^{65}$ What has been learnt from all this is that higher QD densities, and better control on the shape, size and band alignment of the QDs are needed in order to use this technology as efficient absorber in IBSCs.

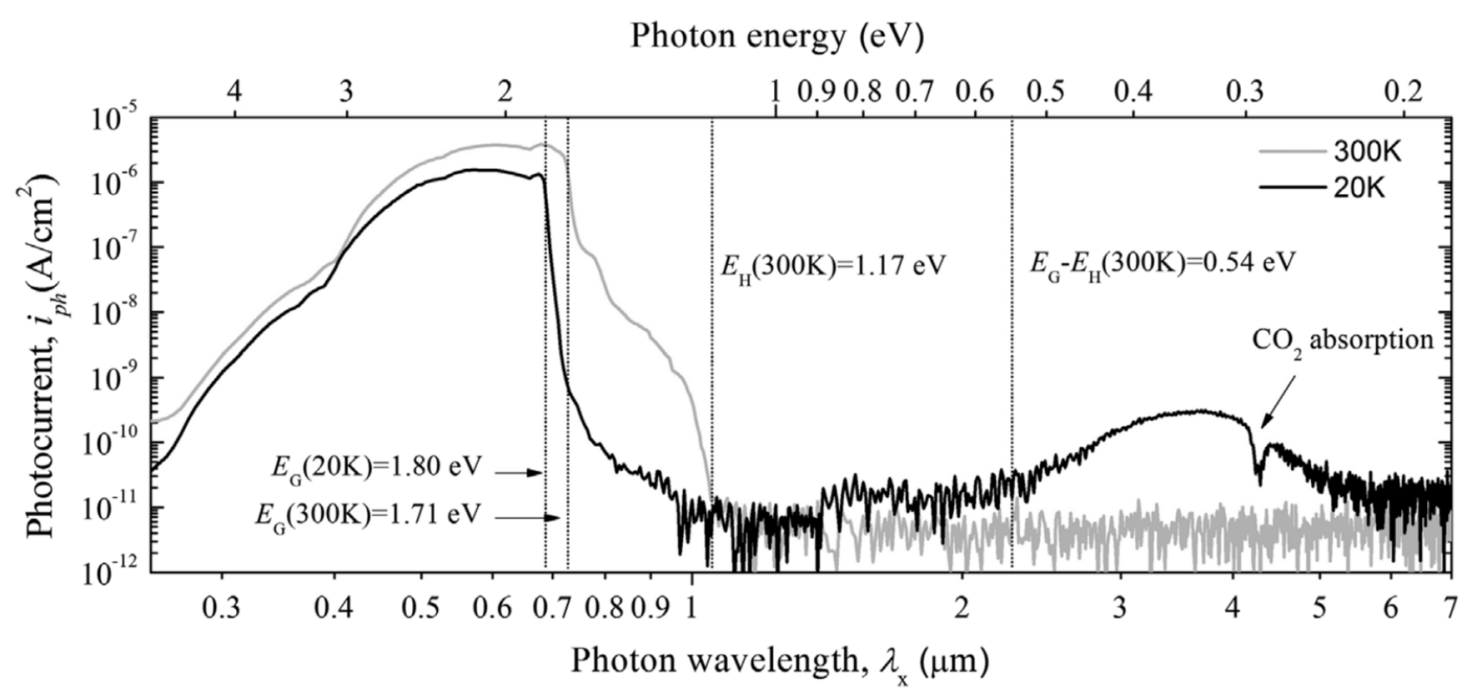

FIGURE 4. Photocurrent measured in an InAs/AlGaAs EQD-based IBSC showing the three absorption thresholds in the IB material. Reproduced with permission from Ref. ${ }^{58}$.

\section{FUTURE DIRECTIONS}

It is difficult to foresee which technology will first succeed in making practical IBSCs. Nonetheless, in this work we want to focus on one kind of QD technology still very little explored in IB devices: colloidal quantum dots (CQDs). CQDs $^{66}$ are quantum dots synthesized via wet chemical routes that produce nanocrystals dispersed in a solvent. We think that this technological approach has the potential to overcome the main limitations found in EQDs. First, CQDs can be densely packed (volumetric densities of $10^{19}-10^{20} \mathrm{~cm}^{-3}$ ) in solid-state films that are highly absorbent in both the VB $\rightarrow$ IB and the IB $\rightarrow$ CB transitions. ${ }^{67}$ Second, the size of the CQDs can be precisely controlled ${ }^{68}$ allowing for a true gap between the IB and the VB and CB. Additionally, CQD thin-films can be fabricated by low-cost solution-processing techniques, such as spin- 
coating or drop casting, which allows envisaging CQD-based IBSCs operating at one sun. CQDs were first suggested as IB materials by Mendes et al. ${ }^{69}$

One key difference between EQDs and CQDs, resulting from their respective fabrication methods, is that EQDs are grown inside a semiconductor host or matrix, whereas CQDs are selfstanding, in the sense that, once deposited on a substrate, they are surrounded by air. However, it has recently been demonstrated ${ }^{70}$ that perovskites and preformed PbS CQDs, combined in solution phase, can produce epitaxially-aligned dots-in-a-matrix heterocrystals. In this work, we will refer to such a material, in a general manner, as colloidal quantum dots in a matrix (CQDM), which have been also suggested as candidates for IBSCs. ${ }^{71}$ Sketches of CQD-based and CQDMbased IBSCs are shown in Figure 5a-b. Their corresponding simplified band diagrams are depicted in Figure 5c-d, where we assume that the dots are $n$-doped such that the confined ground states of their conduction band is partially populated. An analogous alternative case in which the dots are $p$-doped is also possible but is left out of the discussion for simplicity. In CQDs, the ground state of the conduction band of the dots, plays the role of the IB, whereas the ground state of the valence band and the first excited state of the conduction band of the dots play the role, respectively, of the VB and $\mathrm{CB}$ as they are described in Figure 1a. In CQDMs, the CB and the VB are those of the matrix, just as it was the case in EQDs.

Both approaches are, in principle, valid for implementing IBSCs from the point of view of strong photon absorption and control over the band diagram. There is, however, an important difference between CQDs and CQDMs that may tip the scale in favor of the latter. CQD films usually have reduced mobilities as compared to crystalline bulk semiconductors, because transport relies on carrier hopping between neighboring $\operatorname{dots}^{72}$ (see Figure 5c). In this situation, long carrier lifetimes for the $\mathrm{CB} \rightarrow \mathrm{IB}$ recombination would be required to achieve efficient carrier collection. However, evidence in some CQD materials suggests that this lifetime is in the sub-nanosecond regime. ${ }^{73,74}$ To solve this issue, one challenging pathway would be to engineer the CQDs so that they exhibit band-like transport and high mobility ${ }^{75}$ through the CB and the VB. In CQDM-based devices, on the other hand, charge transport occurs naturally within the bands of the crystalline matrix, with higher mobility, thus favoring carrier extraction. Additionally, the CQDM approach allows decoupling the absorption coefficient between the two component materials: the dots need only to be strong absorbers in the two sub-gaps $\left(E_{H}\right.$ and $\left.E_{L}\right)$, whereas the matrix can be a strong absorber for photon energies greater than $E_{G}$. Nevertheless, the number of available different CQDM materials is still limited. ${ }^{76}$ 
(a)

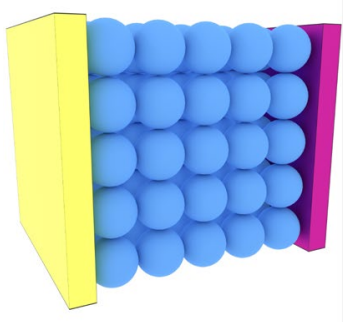

(c)

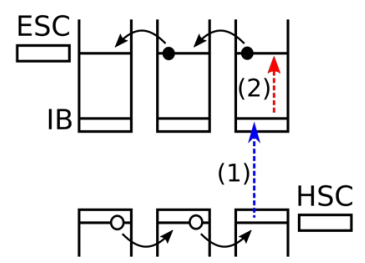

(b)

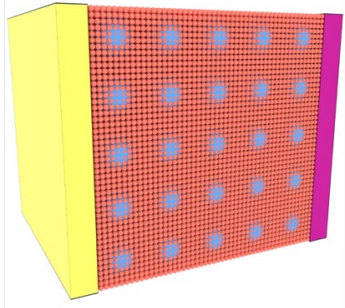

(d)

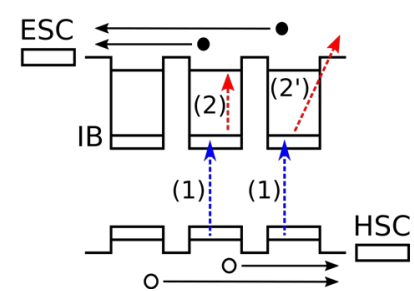

FIGURE 5. Sketches of (a) a CQD-based IBSC and (b) a CQDM-based IBSC. (c) and (d) illustrate the band diagrams of (a) and (b), respectively. In (c) charge transport occurs between confined states of adjacent QDs. In (d) charge transport occurs within the VB and the CB of the matrix. (1) and (2) represent absorption processes between confined states of the QDs, whereas (2') represents absorption between a QD confined state and a delocalized state in the matrix.

The first CQDM-based IBSC prototypes, using PbS CQDs in a perovskite matrix, have provided satisfactory results. ${ }^{77}$ Monochromatic below-bandgap absorption was demonstrated, proving that the IB is optically active in the device (Figure 6b). TPPC was also reported, although it yielded very low currents (Figure 6c). In our opinion, the low values of the TPPC may be due to two main reasons. (i) Absorption from the IB to the CB is proportional to the occupancy of the IB. If the IB is naturally empty of electrons, $\mathrm{IB} \rightarrow \mathrm{CB}$ absorption will be hindered. Hence, it is possible that pre-doping of the CQDs is needed in order to semi-fill the IB, so that both the VB $\rightarrow$ IB and the $\mathrm{IB} \rightarrow \mathrm{CB}$ absorptions are strong. ${ }^{78}$ This represents an additional challenge, since controlling doping in CQDs is not an easy task. ${ }^{79}$ (ii) The experiments performed in Ref. ${ }^{77}$ probe the IB $\rightarrow \mathrm{CB}$ transition as occurring between a confined state of the QDs and the delocalized states of the matrix (transition 2' in Figure 5). Such transition has an energy of around $0.8 \mathrm{eV}$ (Figure 6a). Although this requires further studies, it is possible that the probability of this transition is not very strong. Instead, as discussed earlier, IB $\rightarrow \mathrm{CB}$ absorption can be strong in CQDs if the transition takes place between confined states ${ }^{67,74}$ (transition 2 in Figure 5). However, in the CQDs used in Ref. ${ }^{77}\left(E_{H}=1.0 \mathrm{eV}\right)$, the transition between confined states that would represent $E_{L}$ is smaller than 0.3 $\mathrm{eV} \cdot{ }^{67}$ 
As a guideline for future experiments using CQDM, we think that emphasis must be put in engineering the band alignment of the CQDs and the matrix so that it resembles that of Figure $5 \mathrm{~d}$ (the first excited state of the conduction band of the QDs should be closely aligned with the bottom edge of the $\mathrm{CB}$ of the matrix). This would allow relying on strong absorption between confined states (for below-bandgap photons) and would guarantee a true energy gap between the IB and the bands of the matrix, which would reduce non-radiative recombination. We remark also that, to achieve the highest efficiencies at one sun, values of $E_{L}$ greater than $0.5 \mathrm{eV}$ are required, as it can be deduced from Figure 1b. Therefore, small QDs should be targeted so that the strong quantum confinement allows such energy differences between consecutive confined states.

(a)

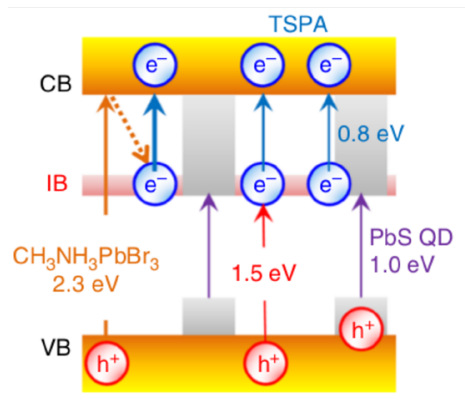

(b)

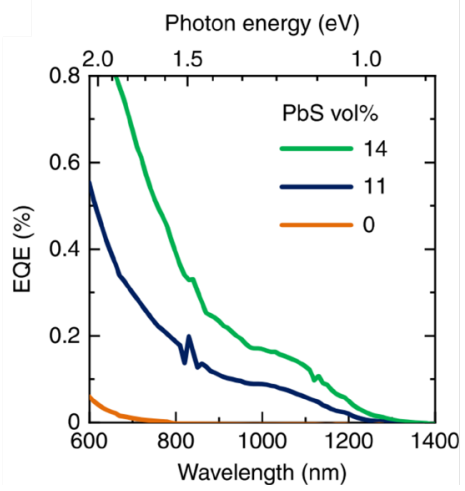

(c)



FIGURE 6. (a) Band diagram and the different absorption thresholds in a PbS/perovskite CQDMbased IBSC. TSPA stands for two-step photon absorption. (b) EQE as a function of the PbS QDs content. (c) Increase in the EQE upon addition of a second beam of IR light. Reproduced from Ref. ${ }^{77}$, licensed under CC BY 4.0.

\section{CONCLUSIONS}

IBSC research has reached a mature state. The theoretical framework is well established and understood thanks to continuous progress in experimentation using four technological IB approaches: QDs, DLIs, HMAs, and OMs. Each technology has its strengths and weaknesses, but overall QDs is the one that has verified most of the phenomena expected in IBSC operation. OMs have potential as a low-cost technology, but their development in IBSCs is still at its infancy. Regarding DLIs and HMAs, we advise the community to focus efforts on understanding the mechanisms of non-radiative recombination introduced by the IB, so that they can be suppressed. Within the QD approach, CQDs have emerged as a technology with potential for overcoming the two main hindrances encountered in EQD-based IBSCs: weak below-bandgap absorption and fast non-radiative recombination between the IB and the VB or the CB. Moreover, CQDs is a 
potentially low-cost technology, which allows envisaging the use of IBSCs in flat plate PV. In this regard, we have discussed how the IBSC concept is still very powerful without sunlight concentration, and we advocate for steering IBSC research towards low cost and high efficiency at one sun.

\section{Acknowledgements}

This work was supported in part by the Project 2GAPS (TEC2017-92301-EXP) funded by the Spanish Ministerio de Ciencia, Innovación y Universidades and the Project MADRID-PV2-CM (P2018/EMT-4308) funded by the Comunidad de Madrid supported with FEDER funds.

\section{REFERENCES}

1. Luque, A. \& Martí, A. Increasing the efficiency of ideal solar cells by photon induced transitions at intermediate levels. Physical Review Letters 78, 5014-5017 (1997).

2. Wolf, M. Limitations and possibilities for improvement of photovoltaic solar energy converters: part I: considerations for earth's surface operation. Proceedings of the IRE $\mathbf{4 8 ,}$ 1246-1263 (1960).

3. Shockley, W. \& Queisser, H. J. Detailed balance limit of efficiency of $\mathrm{p} n$ junction solar cells. Journal of Applied Physics 32, 510-519 (1961).

4. Yoshida, M., Ekins-Daukes, N. J., Farrell, D. J. \& Phillips, C. C. Photon ratchet intermediate band solar cells. Applied Physics Letters 100, 263902-263904 (2012).

5. Pusch, A. \& Ekins-Daukes, N. J. Voltage Matching, Étendue, and Ratchet Steps in Advanced-Concept Solar Cells. Phys. Rev. Applied 12, 044055 (2019).

6. Martí, A. \& Luque, A. Electrochemical Potentials (Quasi-Fermi Levels) and the Operation of Hot-Carrier, Impact-Ionization, and Intermediate-Band Solar Cells. Photovoltaics, IEEE Journal of 3, 1298-1304 (2014).

7. Martí, A. From the Hot Carrier Solar Cell to the Intermediate Band Solar Cell, Passing through the Multiple-Exciton Generation Solar Cell and then back to the Hot Carrier Solar Cell: the Dance of the Electro-Chemical Potentials. in 36th European Photovoltaic Solar Energy Conference and Exhibition 6-12 (2019). 
8. Martí, A. \& Araújo, G. L. Limiting efficiencies for photovoltaic energy conversion in multigap systems. Solar Energy Materials and Solar Cells 43, 203-222 (1996).

9. Ramiro, I., Martí, A., Antolín, E. \& Luque, A. Review of experimental results related to the operation of intermediate band solar cells. IEEE Journal of Photovoltaics 4, 736-748 (2014).

10. Okada, Y. et al. Intermediate band solar cells: Recent progress and future directions. Applied Physics Reviews 2, 21302 (2015).

11. Luque, A. Will we exceed 50\% efficiency in photovoltaics? Journal of Applied Physics 110, 031301 (2011).

12. Louwen, A., van Sark, W. G. J. H. M., Faaij, A. P. C. \& Schropp, R. E. I. Re-assessment of net energy production and greenhouse gas emissions avoidance after 40 years of photovoltaics development. Nat Commun 7, 13728 (2016).

13. Geisz, J. F. et al. Six-junction III-V solar cells with $47.1 \%$ conversion efficiency under 143 Suns concentration. Nature Energy 5, 326-335 (2020).

14. CPV Solar Cells - AZUR SPACE Solar Power GmbH. http://www.azurspace.com/index.php/en/products/products-cpv/cpv-solar-cells. Accessed: 2020-09-17.

15. Krishna, A. \& Krich, J. J. Increasing efficiency in intermediate band solar cells with overlapping absorptions. J. Opt. 18, 074010 (2016).

16. Brown, A. S. \& Green, M. A. Impurity photovoltaic effect: Fundamental energy conversion efficiency limits. Journal of Applied Physics 92, 1329-1336 (2002).

17. Cuadra, L., Martí, A. \& Luque, A. Influence of the overlap between the absorption coefficients on the efficiency of the intermediate band solar cell. Electron Devices, IEEE Transactions on 51, 1002-1007 (2004).

18. López, E., Martí, A., Antolín, E. \& Luque, A. On the Potential of Silicon Intermediate Band Solar Cells. Energies 13, 3044 (2020).

19. Martí, A., Cuadra, L. \& Luque, A. Quantum dot intermediate band solar cell. in 28th Photovoltaic Specialists Conference vol. Conference 940-943 (IEEE, 2000). 
20. Luque, A. \& Martí, A. A metallic intermediate band high efficiency solar cell. Progress in Photovoltaics: Research and Applications 9, 73-86 (2001).

21. Krich, J. J., Halperin, B. I. \& Aspuru-Guzik, A. Nonradiative lifetimes in intermediate band photovoltaics -Absence of lifetime recovery. Journal of Applied Physics 112, 013707 (2012).

22. Shan, W. et al. Band anticrossing in GaInNAs alloys. Physical Review Letters 82, 1221 1224 (1999).

23. Yu, K. M. et al. Diluted II-VI oxide semiconductors with multiple band gaps. Physical Review Letters 91, 246403 (2003).

24. Ekins-Daukes, N. J. \& Schmidt, T. W. A molecular approach to the intermediate band solar cell: The symmetric case. Applied Physics Letters 93, 2-5 (2008).

25. Singh-Rachford, T. N. \& Castellano, F. N. Photon upconversion based on sensitized triplettriplet annihilation. Coordination Chemistry Reviews 254, 2560-2573 (2010).

26. Palacios, P., Aguilera, I., Sánchez, K., Conesa, J. C. \& Wahnón, P. Transition-MetalSubstituted Indium Thiospinels as Novel Intermediate-Band Materials: Prediction and Understanding of Their Electronic Properties. Phys. Rev. Lett. 101, 046403 (2008).

27. Jiang, L. et al. Semiconducting ferroelectric perovskites with intermediate bands via B-site Bi5+ doping. Phys. Rev. B 90, 075153 (2014).

28. Tablero, C. Survey of intermediate band materials based on $\mathrm{ZnS}$ and $\mathrm{ZnTe}$ semiconductors. Solar Energy Materials and Solar Cells 90, 588-596 (2006).

29. Vörös, M., Galli, G. \& Zimanyi, G. T. Colloidal Nanoparticles for Intermediate Band Solar Cells. ACS Nano 9, 6882-6890 (2015).

30. Jibran, M., Sun, X., Wang, B., Yamauchi, Y. \& Ding, Z. Intermediate band solar cell materials through the doping of group-VA elements (N, P, As and $\mathrm{Sb})$ in $\mathrm{Cu}_{2} \mathrm{ZnSiSe}_{4}$. RSC Adv. 9, 28234-28240 (2019).

31. Luque, A. et al. General equivalent circuit for intermediate band devices: Potentials, currents and electroluminescence. Journal of Applied Physics 96, 903-909 (2004). 
32. Marsen, B., Klemz, S., Unold, T. \& Schock, H.-W. Investigation of the Sub-Bandgap Photoresponse in CuGaS2: Fe for Intermediate Band Solar Cells. Progress in Photovoltaics: Research and Applications 20, 625-629 (2012).

33. Wang, W., Lin, A. S., Phillips, J. D. \& Metzger, W. K. Generation and recombination rates at ZnTe: O intermediate band states. Applied Physics Letters 95, 261103-261107 (2009).

34. Simpson, C. et al. An intermediate band dye-sensitised solar cell using triplet-triplet annihilation. Physical Chemistry Chemical Physics 17, 24826-24830 (2015).

35. Martí, A. et al. Production of photocurrent due to intermediate-to-conduction-band transitions: a demonstration of a key operating principle of the intermediate-band solar cell. Physical Review Letters 97, 247701-247704 (2006).

36. Ramiro, I. et al. Two-photon photocurrent and voltage up-conversion in a quantum dot intermediate band solar cell. in 2014 IEEE 40th Photovoltaic Specialist Conference, PVSC $20143251-3253$ (2014).

37. Tamaki, R., Shoji, Y., Okada, Y. \& Miyano, K. Spectrally Resolved Interband and Intraband Transitions by Two-Step Photon Absorption in InGaAs/GaAs Quantum Dot Solar Cells. IEEE Journal of Photovoltaics 5, 229-233 (2014).

38. Antolín, E. et al. Reducing carrier escape in the InAs/GaAs quantum dot intermediate band solar cell. Journal of Applied Physics 108, 064513 1-7 (2010).

39. Luque, A., Martí, A. \& Cuadra, L. Impact-ionization-assisted intermediate band solar cell. Electron Devices, IEEE Transactions on 50, 447-454 (2003).

40. Antolín, E. et al. Advances in quantum dot intermediate band solar cells. in Conference Record of the IEEE Photovoltaic Specialists Conference 65-70 (2010).

41. Ramiro, I. et al. Analysis of the intermediate-band absorption properties of type-II GaSb/GaAs quantum-dot photovoltaics. Physical Review B 96, 125422 (2017).

42. Ekins-Daukes, N. J., Honsberg, C. B. \& Yamaguchi, M. Signature of intermediate band materials from luminescence measurements. in Photovoltaic Specialists Conference. vol. Conference 49-54 (IEEE, 2005). 
43. López, N., Reichertz, L. A., Yu, K. M., Campman, K. \& Walukiewicz, W. Engineering the Electronic Band Structure for Multiband Solar Cells. Physical Review Letters 106, 4 (2011).

44. Lin, Y. H. L. et al. Enhanced sub-bandgap efficiency of a solid-state organic intermediate band solar cell using triplet-triplet annihilation. Energy and Environmental Science 10, $1465-1475$ (2017).

45. Hill, S. P., Dilbeck, T., Baduell, E. \& Hanson, K. Integrated Photon Upconversion Solar Cell via Molecular Self-Assembled Bilayers. ACS Energy Lett. 1, 3-8 (2016).

46. Sheu, J.-K. et al. Photoresponses of manganese-doped gallium nitride grown by metalorganic vapor-phase epitaxy. Applied Physics Letters 102, 71103-71107 (2013).

47. Hu, K. et al. Iron-incorporated chalcopyrite of an intermediate band for improving solar wide-spectrum absorption. Journal of Solid State Chemistry 277, 388-394 (2019).

48. $\mathrm{Hu}$, K. et al. Intermediate Band Material of Titanium-Doped Tin Disulfide for Wide Spectrum Solar Absorption. Inorg. Chem. 57, 3956-3962 (2018).

49. Han, L., Wu, L., Liu, C. \& Zhang, J. Doping-Enhanced Visible-Light Absorption of CH3NH3PbBr3 by Bi3+-Induced Impurity Band without Sacrificing Bandgap. The Journal of Physical Chemistry C acs.jpcc.8b12026 (2019).

50. Khoshsirat, N. et al. Efficiency enhancement of Cu2ZnSnS4 thin film solar cells by chromium doping. Solar Energy Materials and Solar Cells 201, 110057 (2019).

51. Sampson, M. D., Park, J. S., Schaller, R. D., Chan, M. K. Y. \& Martinson, A. B. F. Transition metal-substituted lead halide perovskite absorbers. J. Mater. Chem. A 5, 35783588 (2017).

52. Nematollahi, M. et al. Interpretation of photovoltaic performance of $\mathrm{n}-\mathrm{ZnO}: \mathrm{Al} / \mathrm{ZnS}: \mathrm{Cr} / \mathrm{p}-$ GaP solar cell. Solar Energy Materials and Solar Cells 169, 56-60 (2017).

53. Garcia-Hemme, E. et al. Vanadium supersaturated silicon system: a theoretical and experimental approach. J. Phys. D: Appl. Phys. 50, 495101 (2017).

54. Lee, M.-L., Huang, F.-W., Chen, P.-C. \& Sheu, J.-K. GaN intermediate band solar cells with Mn-doped absorption layer. Sci Rep 8, 8641 (2018). 
55. Sullivan, J. T., Simmons, C. B., Buonassisi, T. \& Krich, J. J. Targeted Search for Effective Intermediate Band Solar Cell Materials. IEEE J. Photovoltaics 5, 212-218 (2015).

56. Ahsan, N. et al. Two-photon excitation in an intermediate band solar cell structure. Applied Physics Letters 100, 172111-172114 (2012).

57. Antolín, E. et al. Intermediate band to conduction band optical absorption in $\mathrm{ZnTeO}$. in Photovoltaic Specialists Conference (PVSC), 2012 38th vol. 4 1-5 (IEEE, 2012).

58. Datas, A. et al. Intermediate band solar cell with extreme broadband spectrum quantum efficiency. Physical Review Letters 114, (2015).

59. Ramiro, I. et al. Three-Bandgap Absolute Quantum Efficiency in $\mathrm{GaSb} / \mathrm{GaAs}$ Quantum Dot Intermediate Band Solar Cells. IEEE Journal of Photovoltaics 7, 508-512 (2017).

60. Smith, B. L. et al. Inverted growth evaluation for epitaxial lift off (ELO) quantum dot solar cell and enhanced absorption by back surface texturing. in 2016 IEEE 43rd Photovoltaic Specialists Conference (PVSC) 1276-1281 (IEEE, 2016).

61. Shoji, Y., Watanabe, K. \& Okada, Y. Photoabsorption improvement in multi-stacked InGaAs/GaAs quantum dot solar cell with a light scattering rear texture. Solar Energy Materials and Solar Cells 204, 110216 (2020).

62. Feng Lu, H. et al. Plasmonic quantum dot solar cells for enhanced infrared response. Appl. Phys. Lett. 100, 103505 (2012).

63. Mellor, A., Luque, A., Tobías, I. \& Martí, A. The feasibility of high-efficiency InAs/GaAs quantum dot intermediate band solar cells. Solar Energy Materials and Solar Cells 130, $225-233$ (2014).

64. Ramiro, I. et al. InAs/AlGaAs quantum dot intermediate band solar cells with enlarged subbandgaps. in Conference Record of the IEEE Photovoltaic Specialists Conference 652-656 (2012).

65. Tomić, S., Martí, A., Antolín, E. \& Luque, A. On inhibiting Auger intraband relaxation in InAs/GaAs quantum dot intermediate band solar cells. Appl. Phys. Lett. 99, 053504 (2011).

66. Alivisatos, A. P. Semiconductor Clusters, Nanocrystals, and Quantum Dots. Science 271, 933-937 (1996). 
67. Ramiro, I. et al. Size- and Temperature-Dependent Intraband Optical Properties of Heavily n-Doped PbS Colloidal Quantum Dot Solid-State Films. ACS Nano 14, 7161-7169 (2020).

68. Murray, C. B., Norris, D. J. \& Bawendi, M. G. Synthesis and Characterization of Nearly Monodisperse CdE $(\mathrm{E}=\mathrm{S}, \mathrm{Se}, \mathrm{Te})$ Semiconductor Nanocrystallites. Journal of the American Chemical Society 115, 8706-8715 (1993).

69. Mendes, M. J. et al. Self-organized colloidal quantum dots and metal nanoparticles for plasmon-enhanced intermediate-band solar cells. Nanotechnology 24, (2013).

70. Ning, Z. et al. Quantum-dot-in-perovskite solids. Nature 523, 324-328 (2015).

71. Sanchez, R. S. et al. Tunable light emission by exciplex state formation between hybrid halide perovskite and core/shell quantum dots: Implications in advanced LEDs and photovoltaics. Science Advances 2, (2016).

72. Guyot-Sionnest, P. Electrical transport in colloidal quantum dot films. Journal of Physical Chemistry Letters 3, 1169-1175 (2012).

73. Pandey, A. \& Guyot-Sionnest, P. Slow Electron Cooling in Colloidal Quantum Dots. Science 322, 929-932 (2008).

74. Deng, Z., Jeong, K. S. \& Guyot-Sionnest, P. Colloidal quantum dots intraband photodetectors. ACS Nano 8, 11707-11714 (2014).

75. Lan, X. et al. Quantum dot solids showing state-resolved band-like transport. Nat. Mater. 19, 323-329 (2020).

76. Ngo, T. T. \& Mora-Seró, I. Interaction between Colloidal Quantum Dots and Halide Perovskites: Looking for Constructive Synergies. J. Phys. Chem. Lett. 10, 1099-1108 (2019).

77. Hosokawa, H. et al. Solution-processed intermediate-band solar cells with lead sulfide quantum dots and lead halide perovskites. Nature Communications 10, 4-6 (2019).

78. Kim, J., Choi, D. \& Jeong, K. S. Self-doped colloidal semiconductor nanocrystals with intraband transitions in steady state. Chemical Communications 54, 8435-8445 (2018).

79. Stavrinadis, A. \& Konstantatos, G. Strategies for the Controlled Electronic Doping of Colloidal Quantum Dot Solids. ChemPhysChem 17, 632-644 (2016). 